Alan H. Daniels, BA

Courtney L. Wilson, BA

Rebecca A. Harrison, MD

Oregon Health \& Science University, Portland, Oregon
The authors acknowledge Alan Hunter, MD, Thomas DeLoughery, MD, Brittany Wilson, MD, and Kevin White, MD.

\section{Hepatitis C-Associated}

Leukocytoclastic Vasculitis with Anticardiolipin Antibodies Causing Penile Necrosis and Deep Venous Thrombosis in the Absence of Cryoglobulinemia

$\mathbf{L}$ eukocytoclastic vasculitis (LCCV) and deep venous thrombosis - (DVT) are uncommon manifestations of hepatitis C and when seen, are usually associated with cryoglobulinemia. The presence of hepatitis C-associated antiphospholipid antibodies (APLAs) such as anticardiolipin antibodies may increase the risk of deep venous thrombosis. Hepatitis C-associated APLAs and LCCV leading to penile necrosis has not previously been reported, to our knowledge.

\section{CASE}

A previously healthy 57 -year-old white man with hepatitis $C$ presented with a 2- to 3-day history of testicular pain and spreading, tender erythema on his left inner thigh. He reported 2 days of testicular and penile swelling and blackening of his penis 1 day prior to admission. He denied feeling ill, fevers, chills, nausea, vomiting, dysuria, hematuria, abdominal, back or penile pain or trauma, unusual sexual practices, or new medications.

His medical history was significant for IV drug use, hepatitis C infection, and hypertension in the remote past. He was in a 2-year monogamous relationship with his female partner and denied any history of sexually transmitted diseases; however, he did report erectile dysfunction over the last few months. He worked as a bartender and reportedly drank 1 glass of wine per night. He denied tobacco or current IV drug use and did occasionally smoke marijuana. His medications included atenolol, hydrochlorothiazide, fish oil, cottonseed oil, and a multivitamin. He denied use of any herbal supplements or erectile dysfunction medications.

On physical exam he did not appear toxic. Vital signs were temperature of $37.3^{\circ} \mathrm{C}$, blood pressure of $155 / 80$, pulse of 100 , and $\mathrm{O}_{2}$ saturation of $97 \%$ on room air. His HEENT, cardiovascular, lung, and abdominal exams were unremarkable. He had a $5-\mathrm{cm}$ indurated, dark, erythematous lesion on his left thigh, surrounded by diffuse tracking erythema, and an erythematous and indurated suprapubic region. His uncircumcised penis was swollen and black, with a sharp demarcation near the base of the shaft (Fig. 1). A CT scan with oral and IV contrast demonstrated thickening and edema of the scrotum, suprapubic soft tissue, and penis, with 


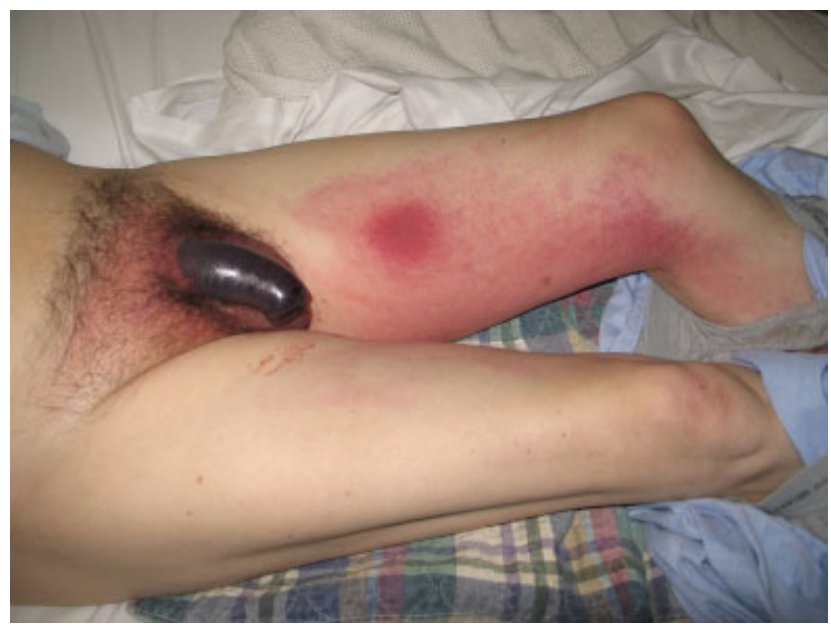

FIGURE 1. Uncircumsised, black, and swollen penis with sharp demarcation near the base of the shaft. Also note the $5 \mathrm{~cm}$, indurated, erythematous lesion on his left thigh, surrounded by diffuse tracking erythema on left leg.

asymmetric enlargement of the left corpora. Mild cirrhosis with associated small gastric varices was also noted. No thrombosis, atherosclerosis, or gas or fluid collection was noted. The bladder, prostate, and seminal vesicles were normal.

A punch biopsy of the leg lesion revealed LCCV with dense fibrin deposition throughout the vessels. Abnormal laboratory data included a mildly elevated WBC count, decreased hemoglobin, thrombocytopenia, mild hyponatremia, low albumin, mildly increased glucose, mild transaminitis, and increased bilirubin. He also had an increased aPTT, elevated ESR, positive hepatitis C PCR and antibodies, positive rheumatoid factor, and high titers of anticardiolipin IgM and anti-B2GPI IgM.

On hospital day 2, a urological surgery was performed to remove the necrotic penile tissue, including the foreskin, down to the spared tunica albuginea. Pathology studies of the tissue specimens revealed highly vascular subcutaneous tissue with hemorrhage and focal denudation, consistent with necrosis. Following surgery, the patient's platelet count and INR returned to normal levels. No steroids or cytotoxic agents were given. On hospital day 6 , the patient developed bilateral leg pain and swelling. Lower extremity doppler ultrasound examination revealed occlusive DVT of the right gastrocnemius, popliteal, and greater saphenous veins, as well thromboses in the left gastrocnemius, soleal, posterior tibial, and greater saphenous veins; thus, enoxaparin therapy was initiated. On hospital day 8 , the patient returned to the operating room for a penile tunneling procedure, in which the penis was surgically inserted into the scrotum as an alternative to skin grafting. He recovered well from the surgeries and was discharged on hospital day 11 on oral anticoagulation with warfarin. At follow-up 1 month after discharge, the patient was doing well and planned for surgery to free his penis from the scrotal sac in 2 months' time.

\section{DISCUSSION}

This case illustrates uncommon extrahepatic manifestations of hepatitis $\mathrm{C}$, including leukocytoclastic vasculitis and deep venous thromboses. Our patient, with abnormal LFTs and positive hepatitis C titers, presented with tissue necrosis of the penis and an unidentifiable erythematous lesion on the leg and subsequently developed multiple deep venous thromboses during his hospital course. Initial diagnostic considerations of the penile and skin lesions included fixed drug reaction, trauma, ischemia, infection, arachnid bite, and vasculitis. The patient denied exposure to NSAIDS, antibiotics, anticonvulsants, or anticoagulants, which are commonly reported causes of fixed drug reactions. He denied trauma or spider or bug bites, was nontoxic appearing, afebrile, and had a near-normal white blood cell count. While awaiting laboratory and biopsy results, we did not initiate pharmacological therapy because of the unknown etiology of the patient's pathology. The patient's workup revealed that his symptoms were most likely secondary to cryoglobulin-negative hepatitis $\mathrm{C}$ infection with leukocytoclastic vasculitis and antiphospholipid antibodies, leading to necrosis of the penile prepuce-an entity that, to our knowledge, has not been reported.

Leukocytoclastic vasculitis is a complication of many diseases including Henoch-Schönlein purpura, Wegener's granulomatosis, sepsis, ANCA-associated vasculitis, SLE, and hepatitis C. ${ }^{1-4}$ Leukocytoclastic vasculitis often presents with palpable purpura but may also present with frank necrosis. ${ }^{4}$ Penile leukocytoclastic vasculitis has been reported in the literature previously ${ }^{5}$; however, most of these cases involve Wegener's granulomatosis and Henoch-Schönlein purpura. One case series demonstrated that approximately $1 \%$ of patients with hepatitis $C$ develop vasculitis during the course of their illness. ${ }^{19}$ There has been 1 reported case of penile leukocytoclastic vasculitis, which occurred in a patient with hepatitis $\mathrm{C}$ who was found to also have cryoglobulinemia. ${ }^{6}$ Our patient tested negative for 
cryoglobulins twice during his hospital stay and also had normal complement levels, which strongly weighs against cryoglobulinemia. One study reported that up to $75 \%$ of patients with hepatitis C who develop leukocytoclastic vasculitis will test positive for cryoglobulins ${ }^{6}$; thus, our patient's presentation with cryoglobulin-negative leukocytoclastic vasculitis is rare.

Our patient also had a positive titer of anticardiolipin antibodies, which are a subset of APLAs. Antiphospholipid antibodies can be found in autoimmune disease, acute and chronic viral infections, and malignancy. ${ }^{7,8}$ Furthermore, APLAs can manifest with arterial and venous thrombosis, and up to $33 \%$ of patients with hepatitis C test positive for APLAs. ${ }^{7}$ The etiology and thrombogenicity of these autoantibodies in the setting of chronic viral hepatitis is still largely unknown, but it has been hypothesized that APLAs may be an autoimmune manifestation of hepatitis $\mathrm{C}$.

Our patient also tested positive for anti-beta2glycoprotien-1 antibodies, the presence of which may be associated with the occurrence of thrombotic events. ${ }^{9}$ The presence of these antibodies strengthens the likelihood that this patient's APLAs were pathogenic and likely associated with his skin necrosis as well as his numerous venous thromboses. Previously documented thromboses in patients with hepatitis $\mathrm{C}$ and APLAs include avascular bone necrosis, venous thromboembolism, MI, stroke, and cutaneous necrosis. ${ }^{10}$ There was 1 reported case of a patient with HIV and anticardiolipin antibodies with cutaneous necrosis and testicular thrombosis, ${ }^{10}$ however, to our knowledge there have been no reported cases of penile necrosis in association with APLAs in a patient with hepatitis C. In this case, treatment with steroids or cytotoxic agents was not warranted because of insufficient evidence to support this practice. However, lifelong anticoagulation with moderate-intensity warfarin to prevent future thrombosis is indicated. ${ }^{11}$ These antibodies and their treatment are poorly understood, and further studies are needed to gain insight into both their development and their role in the pathogenesis of disease in patients with viral hepatitis.

In summary, this patient experienced devastating complications of chronic hepatitis $\mathrm{C}$ infection, leading to necrosis of the penile prepuce and multiple venous thromboses. This case demonstrates that extrahepatic symptoms of hepatitis $\mathrm{C}$ infection, including skin manifestations secondary to leukocytoclastic vasculitis with or without cryglobulinemia, may occur. Furthermore, this case illustrates the increased risk of thrombosis and cutaneous necrosis in patients with chronic hepatitis $C$ infection and associated antiphospholipid antibodies.

Address for correspondence and reprint requests: Rebecca A. Harrison, MD, Oregon Health \& Science University, Department of Hospital Medicine, 3181 SW Sam Jackson Park Rd., BTE 119, Portland OR 97239; Fax: (503) 494-1159; E-mail address: harrisor@ohsu.edu

Received 18 March 2007; revision received 19 June 2007; accepted 7 July 2007.

\section{REFERENCES}

1. Schwaber MJ, Zlotogorski A. Dermatologic manifestations of hepatitis C infection. Int J Dermatol. 1997;36:251-254.

2. Ramos-Casals M, Font J. Extrahepatic manifestations in patients with chronic hepatitis C virus infection. Curr Opin Rheumatol. 2005;17:447-455.

3. Dervis E, Serez K. The prevalence of dermatologic manifestations related to chronic hepatitis $C$ virus infection in a study from a single center in Turkey. Acta Dermatovenerol Alp Panonica Adriat. 2005;14:93-98.

4. Sunderkotter C, Bonsmann G, Sindrilaru A, Luger T. Management of leukocytoclastic vasculitis. J Dermatolog Treat. 2005;16:193-206.

5. Mendéz P, Saeian K, Reddy R, et al. Hepatitis C, cryoglbulinemia, and cutaneous vasculitis associated with unusual and serious manifestations. Am J Gastroenterol. 2001;96: 2489-2493.

6. Cacoub P, Poynard T, Ghillani P, et al. Extrahepatic manifestations of chronic hepatitis C. Arthritis Rheumatism. 1999;42:2204-2212.

7. Biron C, Andreani H, Blanc, P. Prevalence of antiphospholipid antibodies in patients with chronic lever disease related to alcohol or hepatitis $C$ virus: correlation with liver injury. J Lab Clin Med. 1998;31:243-250.

8. Khamashta MA, Hughes GRV. Antiphospholipid antibodies and antiphospholipid syndrome. Curr Opin Rheumatol. 1995;7:389-94.

9. Yuste JR, Prieto J. Anticardiolipin antibodies in chronic viral hepatitis. Do they have clinical consequences? Eur J Gastroenterol Hepatol. 2003;15:717-719.

10. Ramos-Casals M, Cervera R, Lagrutta M, et al. Clinical features related to antiphospholipid syndrome in patients with chronic viral infections (hepatits $\mathrm{C}$ virus/HIV infection): description of 82 cases. CID. 2004;38:1009-1016.

11. Lim W, Crowther MA, Eikelboom JW. Management of antiphospholipid antibody syndrome: A systematic review. JAMA. 2006;295:1050-1057. 